\title{
Kinetics of Hydrogen Desorption from Rapidly Solidified Al-Cr Alloys*
}

\author{
Iya I. Tashlykova-Bushkevich ${ }^{1}$, Goroh Itoh $^{2}$, Vasiliy G. Shepelevich ${ }^{3}$ and Takahiro Shikagawa \\ ${ }^{1}$ Department of Physics, Belarusian State University of Informatics and Radioelectronics, Minsk 220013, Belarus \\ ${ }^{2}$ Department of Mechanical Engineering, Ibaraki University, Hitachi 316-8511, Japan \\ ${ }^{3}$ Department of Solid Physics, Belarusian State University, Minsk 220050, Belarus \\ ${ }^{4}$ Graduate Student, School of Science and Engineering, Ibaraki University, Ibaraki 316-8511, Japan
}

\begin{abstract}
The thermal desorption spectroscopy has been applied to analyse hydrogen desorption from foils of Al-Cr alloys containing up to 3.0 mol\% $\mathrm{Cr}$ produced by centrifugal melt quenching. Surface morphology of the alloys was monitored using atomic force microscopy and scanning electron microscopy. It was revealed that hydrogen behaviour is strongly affected by microstructural features available due to rapid solidification and represents at least four hydrogen trap sites in $\mathrm{Al}-\mathrm{Cr}$ alloys. The $\mathrm{Cr}$ atoms in lattice sites are identified as predominant trap site. The occupancy of dislocations was estimated to be rather high in contrast to vacancies and pores in alloys. The amount of hydrogen trapped by vacancies is drastically decreased with increase in $\mathrm{Cr}$ concentration. These hydrogen/microstructure interactions were discussed regarding rapidly solidified pure aluminum as well as traditionally processed aluminum samples. [doi:10.2320/matertrans.L-MZ201104]
\end{abstract}

(Received October 2, 2010; Accepted December 29, 2010; Published April 13, 2011)

Keywords: thermal desorption spectroscopy, rapid solidification, aluminum-chromium alloys, hydrogen

\section{Introduction}

Nowadays development of embrittlement-resistant materials is considered to be one of the most technically challenging aspects of hydrogen storage in innovative fuel cell vehicles. Although considerable effort has been made to explain the phenomenon of hydrogen embrittlement in metals and alloys, ${ }^{1-3)}$ it has been recognised that the mechanisms associated with such degradation in mechanical properties remain incompletely understood from the mechanistic point of view. Since recently aluminium alloys have been regarded as lightweight materials for high pressure gas storage $\operatorname{tank}^{4,5)}$ basic research needs related to the problem of their hydrogen embrittlement include such issues as hydrogen behaviour and its interaction with lattice defects (vacancies, pores, solute impurities, grain boundaries, dislocations, etc.). Up to date, numerous investigations are conducted on traditionally processed aluminium materials and highlight the key role of the microstructure in hydrogen trapping and segregation. ${ }^{6-9)}$ What has been lacking, so far, is identification in aluminum based alloys of hydrogen interactions with enhanced microstructure solidified at exceptionally high cooling rates. At the same time, effect of such advanced technique as rapid solidification processing (RSP) on the microstructure of aluminium materials yields desirable improving of properties in comparison with conventionally processed counterparts ${ }^{10,11)}$ and includes extended solid solution range, refinement of as-solidified microstructure, reduced levels of segregation, formation of non-equilibrium phases as well as a high density of defects (vacancies and dislocations) in the metal. ${ }^{11}$ ) Therefore analysis of hydrogen behaviour in rapidly solidified (RS) aluminum alloys is of considerable importance because of its potential impact on developing mechanistic understanding of hydrogen/microstructure interactions in order to

*The Paper Contains Partial Overlap with the ICAA12 Proceedings by USB under the Permission of the Editorial Committee. control hydrogen embrittlement in high strength aluminum based materials.

This paper focuses on the investigation of hydrogen trapping in $\mathrm{RS}$ foils of Al-Cr alloys prepared through centrifugal melt quenching. Note that RS alloys on basis of $\mathrm{Al}-\mathrm{Cr}$ system are an example of heat-resistant lightweight structural materials because of their high-temperature mechanical properties and enhanced thermal stability. ${ }^{12,13)}$ In the study, thermal desorption spectroscopy (TDS) was applied to detect hydrogen evolution from RS alloys. In fact, hydrogen is the only gas that is appreciably soluble in aluminium and its alloys. Difficulties in detection of hydrogen states in the materials are mainly caused by its high mobility and small quantity. The thermal desorption analysis represents a modern approach to this problem and can be regarded as a superior technique because of its sensitivity and the accuracy of measurements. ${ }^{14,15)}$ To monitor surface morphology of the foils we employed atomic force microscopy (AFM) and scanning electron microscopy (SEM). It should be noticed that initial research ${ }^{16,17)}$ on $\mathrm{H}$ desorption processes in $\mathrm{RS}$ aluminum and preliminary results obtained for its lightly doped RS binary alloys with $\mathrm{Ti}, \mathrm{Cr}$ and $\mathrm{Zr}$ have clearly demonstrated a great difference between hydrogen behavior in RS aluminum foils and one in traditionally processed aluminum samples. ${ }^{7,18)}$ Therefore the purpose of the present research is to examine hydrogen desorption kinetics in $\mathrm{RS}$ $\mathrm{Al}-\mathrm{Cr}$ alloys in relation to their microstructural features depending on alloy composition. The identification of $\mathrm{H} /$ microstructure interactions in the Al-Cr alloys was discussed in contrast to aluminum prepared through RSP as well as traditionally processed samples.

\section{Experimental}

The Al-Cr alloys with Cr content (mol\%) of 1.0, 1.5 and 3.0 were manufactured from $99.99 \%$ pure aluminum and 99.9\% pure $\mathrm{Cr}$ by melting in an induction electric furnace under an argon atmosphere. Specimens of the alloys were 

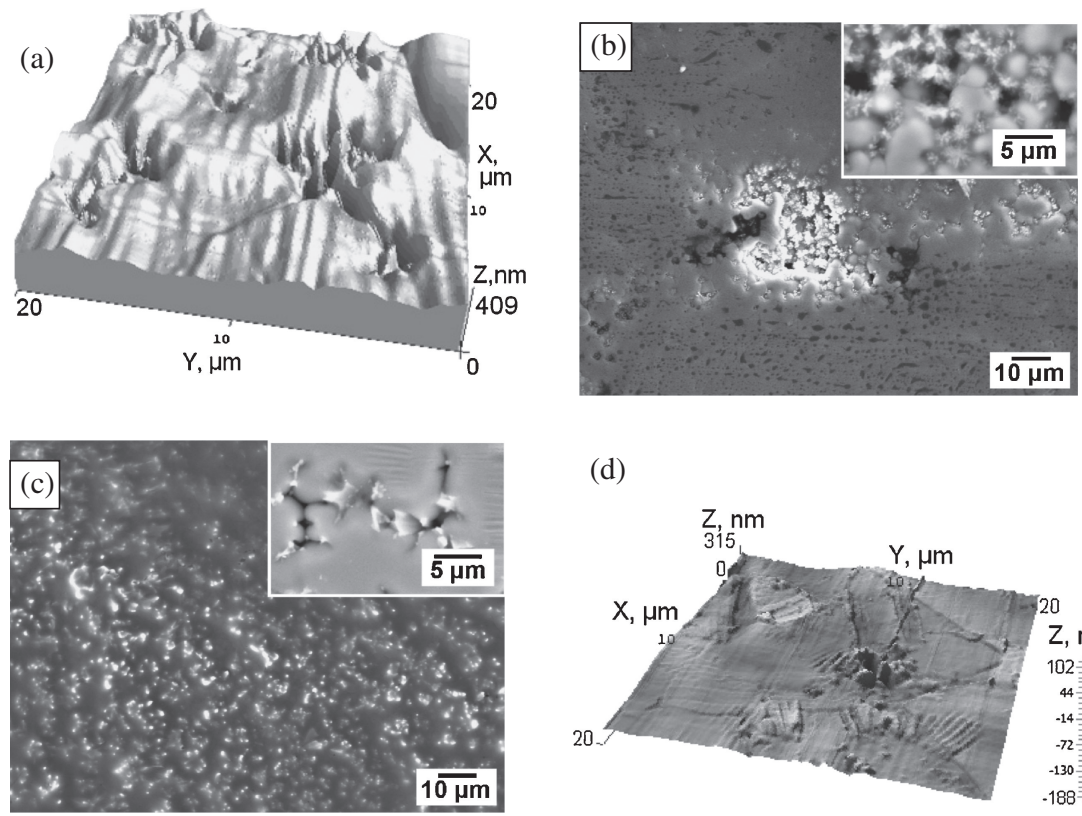

(d)

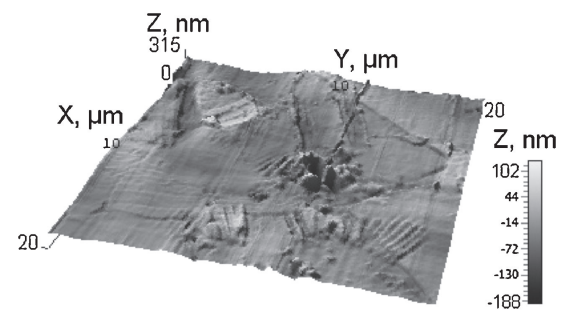

Fig. 1 Typical AFM and SEM observation of RS Al-Cr alloys. (a) the drum-side of as-cast Al-1.0 Cr alloy foil, (b) the drum-side and (c) air-side of as-cast Al-3.0 Cr alloy foils, (d) the drum-side of TDS tested $\mathrm{Al}-1.0 \mathrm{Cr}$ alloy foil.

then rapidly solidified by centrifugal melt quenching at a cooling rate of the order of $10^{6 \circ} \mathrm{C} \cdot \mathrm{s}^{-119)}$ as follows. The droplet of molten alloy was splashed in the air on the inner surface of the polished copper drum with a diameter of $20 \mathrm{~cm}$, rotating at $16 \mathrm{~m} \cdot \mathrm{s}^{-1}$ liner velocity. This produced microcrystalline foils, typically up to $8 \mathrm{~cm}$ in length, 30 $60 \mu \mathrm{m}$ in thickness and $5-10 \mathrm{~mm}$ in width. ${ }^{20)}$ Hydrogen detected in the present work was the immanency impurity which was mixed in the material during melting and RSP.

As-cast foils of Al-Cr alloys were characterized by means of SEM and AFM techniques. The SEM observations were carried out using a LEO1455 scanning electron microscope operated at $20 \mathrm{kV}$. The AFM images were acquired through an NT-206 instrument. No special treatment was applied to the surface of the samples prior to inspection in a contact mode in air using a Si probe. Height images $\left(20 \times 20 \mu \mathrm{m}^{2}\right.$ area) were collected simultaneously and analysed using the Surface Xplorer and Surface View software. ${ }^{21)}$ Roughness values in this paper refer to the average surface roughness values, $R_{\mathrm{a}}$, calculated from AFM surface topographic data.

Hydrogen trapping and evolution were evaluated in TDS measurements employing an ESCO EMD-WA1000S/W machine described in detail elsewhere. ${ }^{22)}$ Generally, TDS curve describes a hydrogen evolution from the sample upon heating by plotting the rate of hydrogen desorption versus temperature. It enables to characterize the types of multiple traps for hydrogen by detecting of temperatures $T_{\mathrm{m}}$ associated with desorption peaks for each trap state. Each observed desorption maximum depends on the activation energy for hydrogen release from the trap as well as the content of trapped hydrogen and the trap density. Before examination, the RS specimens were sectioned. Sample of 6-12 mg from each foil was etched in 10\% sodium hydroxide (1 min), 10\% nitric acid $(1 \mathrm{~min})$, rinsed in water, dried, and immediately tested through TDS technique. During a TDS test, a foil was heated from 120 to $600^{\circ} \mathrm{C}$ at a constant heating rate $\beta$ of $20^{\circ} \mathrm{C} \cdot \mathrm{min}^{-1}$ in a vacuum. To collect background hydrogen spectrum the sample was re-heated under the same conditions when its temperature was reduced to room temperature. Each hydrogen thermal desorption spectrum was plotted with Origin Pro software ${ }^{23)}$ subtracting the background. The TDS treated samples were further analyzed by using AFM.

\section{Results and Discussion}

The AFM was utilized to examine surface morphology and roughness of as-cast RS foils of Al-Cr alloys. Representative height mode scans taken from the drum-side surface of the Al-1.0 Cr alloy foils are illustrated in Fig. 1. The gross morphological feature of the foils is wave-like bending of the surface. The roughness value $R_{\mathrm{a}}$ is determined to be $38.5 \mathrm{~nm}$. The height of some "waves" and peaks on the drum-side surface reaches $320 \mathrm{~nm}$, Fig. 1(a). In Fig. 1, SEM micrographs of both surfaces of Al-3.0 Cr alloy show no significant difference in microstructures of the drum-side and air-side surfaces, meaning little difference in quenching effect between both surfaces. The other noticeable microstructural feature is the presence of pores that were not distributed evenly across the foil surface as indicated both by the AFM imaging and SEM imaging in Fig. 1(a), (b) and (c). Figure 1(d) represents that TDS tests resulted in the foil surface smoothing and pores disappearance. The surface roughness value of Al-1.0 $\mathrm{Cr}$ alloy is reduced by a factor of $\sim 3$ after TDS experiments.

Typical TDS spectra from as-cast RS Al-Cr alloys are presented in Fig. 2(a). The data of recognized peak temperatures $T_{\mathrm{m}}$ and trap sites assigned from literature ${ }^{7,17,18,24,25)}$ as discussed later are summarized in Table 1. Note that TDS spectrum from RS foil of pure aluminum ${ }^{17)}$ shown also in Fig. 2(a) for comparison exhibits three distinct desorption peaks at about 270,390 and $490^{\circ} \mathrm{C}$, denoted 1,2 and 3 . 

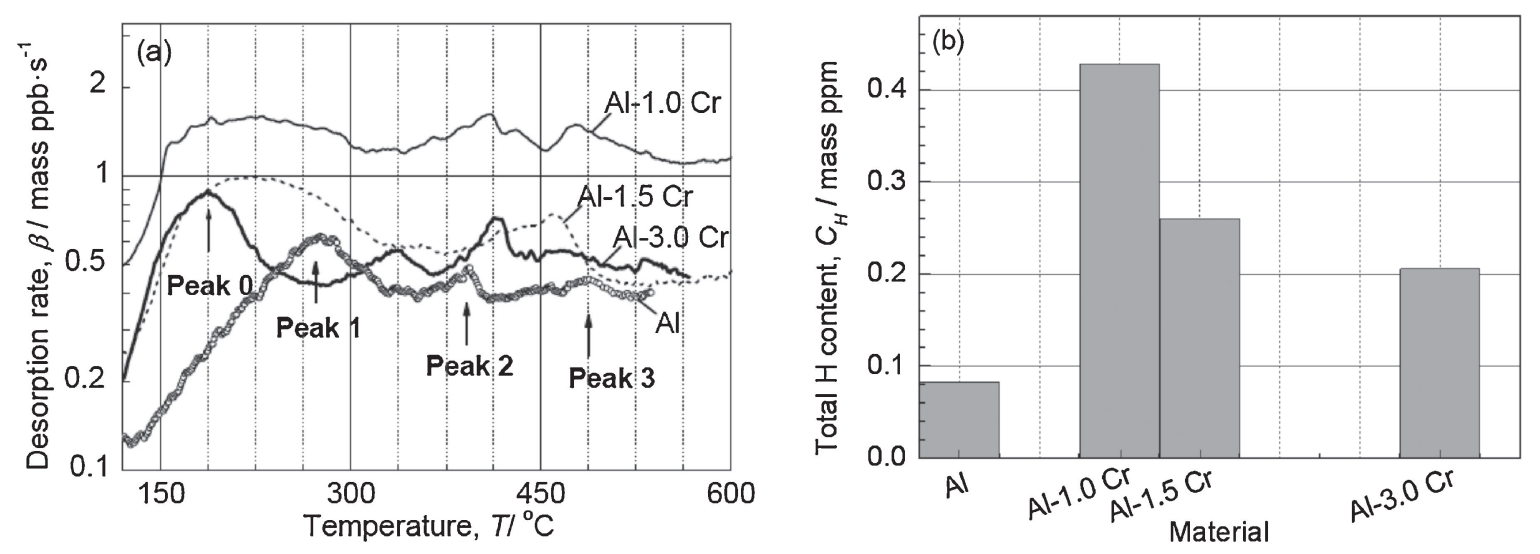

Fig. 2 TDS analysis of RS aluminum (Al) with different $\mathrm{Cr}$ content (mol\%). (a) kinetic curves of hydrogen desorption from foils, taken at a heating rate of $20^{\circ} \mathrm{C} \cdot \mathrm{min}^{-1}$, (b) histogram showing calculated from (a) hydrogen content in foils. The TDS plot for $99.99 \%$ pure aluminum foil, ${ }^{17)}$ taken at a heating rate of $20^{\circ} \mathrm{C} \cdot \mathrm{min}^{-1}$, is also shown in the Fig. 2(a) for comparison (open circle).
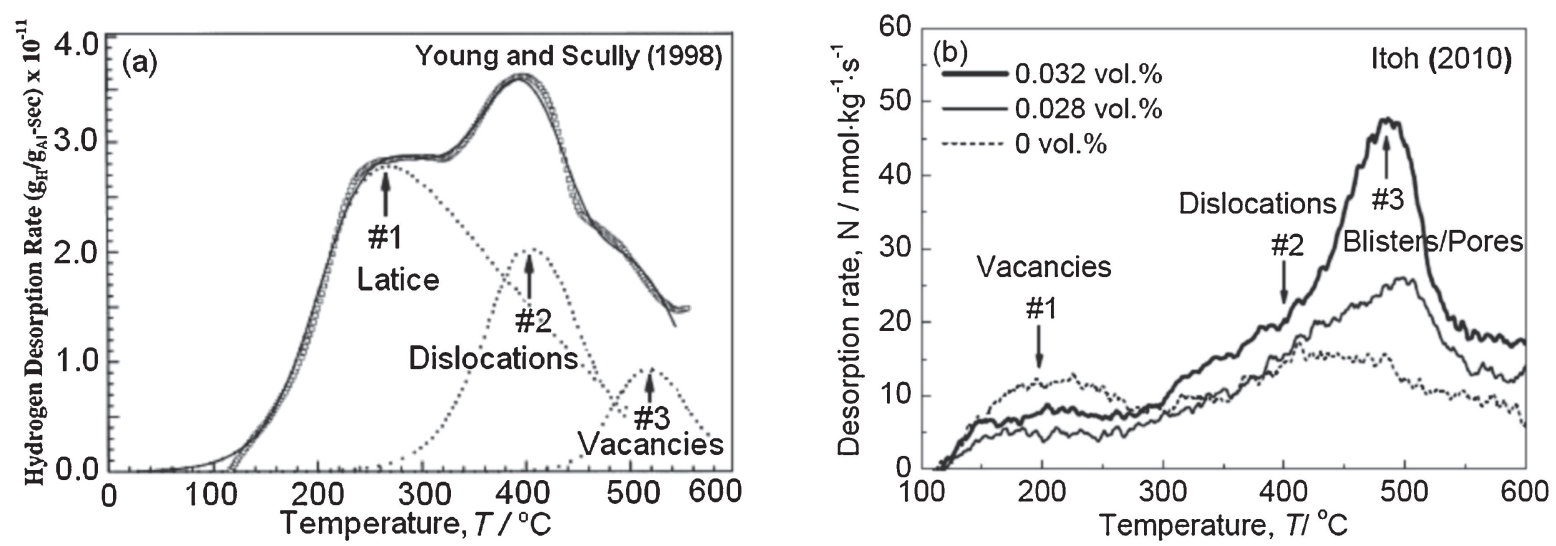

Fig. 3 TDS analysis of traditionally processed aluminum samples. (a) kinetic curve of hydrogen desorption from $99.999 \%$ pure aluminum sample, taken at a heating rate of $10^{\circ} \mathrm{C} \cdot \mathrm{min}^{-1}$, after Young and Scully, ${ }^{18)}$ (b) kinetic curves of hydrogen desorption from $99.99 \%$ pure aluminum specimens with different volume fraction of blisters, taken at a heating rate of $8^{\circ} \mathrm{C} \cdot \mathrm{min}^{-1}$, after Itoh. ${ }^{7}$

Table 1 Trap site identification for hydrogen in RS aluminum (Al) with different $\mathrm{Cr}$ content (mol\%).

\begin{tabular}{lccccc}
\hline \multirow{2}{*}{ Trap site } & \multicolumn{4}{c}{ Peak temperature for trap site, $T_{\mathrm{m}} /{ }^{\circ} \mathrm{C}$} & \multirow{2}{*}{ Trap site description } \\
\cline { 2 - 5 } & $\mathrm{Al}$ & $\mathrm{Al}-1.0 \mathrm{Cr}$ & $\mathrm{Al}-1.5 \mathrm{Cr}$ & $\mathrm{Al}-3.0 \mathrm{Cr}$ & \\
\hline Peak 0 & - & 210 & 210 & 190 & Solute atoms ${ }^{24,25)}$ \\
\hline Peak 1 & 270 & 270 & 270 & 270 & Vacancies $^{7,17)}$ \\
\hline Peak 2 & 390 & 410 & 410 & 410 & Dislocations $^{7,18)}$ \\
\hline Peak 3 & 490 & 480 & 460 & 470 & Pores $^{7,24)}$ \\
\hline
\end{tabular}

Additional prominent low-temperature peak detected at $190^{\circ} \mathrm{C}$ is observed exceptionally in the samples alloyed with $\mathrm{Cr}$ and labeled as 0 on TDS curve from Al-3.0 Cr alloy. It was found out, for the temperature range from 350 to $600^{\circ} \mathrm{C}$, variation in temperatures $T_{\mathrm{m}}$ of desorption peaks is marginal for RS Al-Cr alloys and RS aluminum foils. Overall, for Al1.0 $\mathrm{Cr}$ and $\mathrm{Al}-1.5 \mathrm{Cr}$ alloys, it can be assumed that broad desorption peak lying between 150 and $300^{\circ} \mathrm{C}$ is a superposition of above-indicated peaks 0 and 1 . The low-temperature peak 0 overlaps with a separate peak positioned near $270^{\circ} \mathrm{C}$ in the TDS plot from aluminum. Since desorption rate peaks in TDS spectra have varying breadth and height, it is well known that overlapped and partially hidden peaks are typical for $\mathrm{H}$ trapping at multiple states in a complex microstructure. Therefore it is considered that at least four peaks are identified in the spectra for all Al-Cr alloys, see Table 1. Note that hydrogen thermal desorption curves from RS Al-0.4 Cr alloy ${ }^{16)}$ exhibit peak temperatures identical with those stated above. A new small peak detected at $340^{\circ} \mathrm{C}$ only in the TDS profile from Al-3.0 $\mathrm{Cr}$ alloy is not denoted in Table 1. Figure 2(b) shows the amount of hydrogen $C_{H}$ desorbed from the foils within the examined temperature range, obtained by integrating the spectra plotted in Fig. 2(a). As illustrated in Fig. 2(b), it was observed that total $\mathrm{H}$ egress changed inversely to increase in $\mathrm{Cr}$ content for studied aluminum alloys. Finally, the total concentration of hydrogen $C_{H}$ evolved upon TDS experiments was measured to be 0.206 mass ppm for Al-3.0 Cr alloy.

In most aluminium alloys, transition metals ( $\mathrm{Ti}, \mathrm{Cr}, \mathrm{Fe}, \mathrm{Zr}$, etc.) are known to be employed to increase the thermal stability of conventional alloys because they retard recrystallization. ${ }^{13,26)}$ However, the concentration of these alloying elements are strongly limited and often do not exceed $0.1 \mathrm{~mol} \%$ due to the formation of intermetallic compounds which reduce plasticity, deformability and strength. In our previous work ${ }^{27)}$ it was reported that alloys containing $\mathrm{Cr}$ 
under $1.0 \mathrm{~mol} \%$ produced through centrifugal melt quenching are composed of metastable $\alpha$-solid solution stable up to $300^{\circ} \mathrm{C}$. The presence of intermetallic $\mathrm{Al}_{7} \mathrm{Cr}$ phase particles distributed homogenously in the $\alpha$-aluminium matrix was reported for RS Al-1.5 Cr alloy. ${ }^{27)}$ The average grain size of the RS foils was in the range of several $\mu \mathrm{m}$. According to the present analysis, surface microgeometry characteristics include ridge-rich surface regions and pores in RS Al-Cr alloys. In fact, similar surface topography structures have been already observed for as-cast RS aluminum and Al-Fe alloys. ${ }^{17,28)}$ It was obtained that roughness of foil surface depends on both composition of the sample and topography of contact area of the melt and the drum. As for detected pores, hydrogen porosity in aluminium alloys can be attributed both to solidification shrinkage and gas bubbles expelled from the molten material during RSP. ${ }^{29)}$ From Fig. 1, it is apparent that the heat treatment during TDS tests results in the foil surface smoothing and pore disappearance in part. A similar tendency has been also reported recently for RS pure aluminum. ${ }^{17)}$

In this study, we performed the TDS measurements to reveal the roles of unique microstructural features on the hydrogen desorption and its composition dependence in $\mathrm{RS}$ Al-Cr alloys. It should be pointed out that dislocations, vacancies, lattice interstices (hydrogen in solution), pores, precipitates of secondary phases, solute atoms and grain boundaries are potential trap sites for hydrogen in aluminium and its alloys. Meanwhile, hydrogen interactions with dislocations as well as vacancies are considered to contribute mostly to hydrogen embrittlement of aluminium materials. ${ }^{3,9,25,30)}$ The scope of this paper did not allow for a detailed comparison of the hydrogen-desorption-rate curves from RS pure aluminum with those reported for traditionally processed pure aluminum although references to the hydrogen tapping and evolution in conventional aluminium have been alluded to in the text. Figure 3 exemplifies thermal desorption spectra from pure polycrystalline aluminum ${ }^{18)}$ as well as aluminum produced by a DC cast rolling ingots. ${ }^{7)}$ It is clearly shown that similar to bursts of hydrogen egress from RS pure aluminum demonstrated here in Fig. 2(a), traditionally processed aluminum specimens exhibit three desorption peaks in TDS spectra. The temperatures of peaks are consistent with data listed in Table 1. However, there is controversy about identification of hydrogen trapping in high purity aluminum. In Fig. 3(a) shown that Young and Scully ${ }^{18)}$ have suggested that these trapping sites stated from low to high desorption energy are associated with interstitial lattice sites, dislocations, and vacancies, respectively. Unfortunately, this identification has not helped to clarify results gained by the present authors for RS samples. The fact is that, firstly, this trap identification does not take into account the porosity which is one of the noticeable defects in aluminium alloys. Although RSP reduces porosity of the samples in comparison with casting, ${ }^{29)}$ the pores are easily observed for $\mathrm{RS}$ pure aluminum ${ }^{17)}$ as well as for RS Al-Cr alloys, see Fig. 1. Secondly, it fails to explain our thermal desorption experiments on aluminum foils exposed to humid air after TDS tests. ${ }^{17)}$ On the other hand, recently Itoh ${ }^{7,31)}$ has revealed that these desorption peaks 1,2 and 3 are related to the hydrogen in aluminum trapped by vacancies, dislocations and pores/blisters, respectively, see Fig. 3(b). The vacancy/ hydrogen pairs was deduced to migrate sufficient distance to be released from the inside of the specimen at much lower temperature than the 3rd peak temperature. Furthermore, Toda et $a .^{24,32)}$ have elucidated presence of hydrogen micropores in pure aluminum and its alloys processed through casting as well as hot and cold rolling. It was pointed out for $\mathrm{Al}-\mathrm{Mg}$ alloys that micropores filled and pressurized with molecular hydrogen are predominant hydrogen trap site, while the occupancy for dislocation traps is very high both in the alloys and pure aluminum. ${ }^{24)}$

Therefore it is considered that in RS pure aluminum foils hydrogen resides at trapping sites at vacancies, dislocations and pores as listed in Table 1 . Whereas outward quantitative resemblance of peak positions in TDS profiles, it should be emphasized that quenched-in vacancies play a dominant role in $\mathrm{H}$ trapping in pure aluminum foils produced by means of RSP. Indeed, RS samples exhibit a significant increase of the amount of hydrogen trapped at vacancies instead of pronounced hydrogen trapping at dislocations specified for traditionally processed aluminum specimens, ${ }^{7,18,24)}$ see Figs. 2(a) and 3. Thus, we associate the 270 and $410^{\circ} \mathrm{C}$ trap states as well as the highest binding energy trap site at $470^{\circ} \mathrm{C}$ in $\mathrm{RS} \mathrm{Al-Cr}$ alloys to the same microstructural features as in RS pure aluminum. The trap site having the lowest binding energy (see peak 0 in the TDS spectra) is tentatively attributable to hydrogen in the interstitial lattice sites trapped by $\mathrm{Cr}$ atoms from literature. ${ }^{24,25)}$ The shifting of the first desorption peak in case of $\mathrm{RS} \mathrm{Al}-\mathrm{Zr}$ alloys to a lower temperature compared with $\mathrm{RS}$ pure aluminum provides further evidence and was determined recently. ${ }^{16)}$ It should be noticed, apart from above, the peak 3 may correspond to the $\mathrm{Al}_{7} \mathrm{Cr}$ phase particle coarsening as well as pore disappearance in the alloys. However, the noticeable peak at $480^{\circ} \mathrm{C}$ reported for RS Al-0.4 $\mathrm{Cr}$ alloy ${ }^{16)}$ supports identification of peak 3 as a pore trap state. This fact is evident because of absent of the secondary phase precipitates in RS Al-0.4 Cr alloy foils. ${ }^{27)}$ The identification of the small peak in the vicinity of peak 2 cannot be explained due to a lack of experimental data for Al-Cr alloys and is a subject of further investigations.

This work demonstrates that hydrogen behaviour in RS Al-Cr alloys strongly depends on microcrystalline structure and exhibits composition dependence. As seen in Fig. 2, hydrogen desorption kinetics in the foils are qualitatively changed in the presence of alloying element. The $\mathrm{Cr}$ atoms in lattice sites are identified as predominant hydrogen trap site. The amount of hydrogen trapped at vacancies in the alloys is obviously decreased with increase in $\mathrm{Cr}$ content and with the formation of vacancy-solute atom complexes. ${ }^{33)}$ In fact, vacancies play an important role in the transport of alloying element atoms even during $\mathrm{RSP}^{34)}$ and cause nonuniform dope depth distribution in the near-surface region of RS aluminum alloy foils. ${ }^{33)}$ It also can be inferred that the amount of $\mathrm{H}$ associated with dislocations increases with increasing $\mathrm{Cr}$ content in the foils. In contrast, along with increase in Cr-rich precipitates the role of pores in $\mathrm{H}$ trapping is decreased in the alloys. Meanwhile, height of the desorption peak related to hydrogen release from pores exceeds the one in case of RS pure aluminum and can be 
caused by known fact that aluminium alloys usually show higher porosity than high-purity aluminium. ${ }^{24)}$ The total $\mathrm{H}$ egress obtained was changed inversely to $\mathrm{Cr}$ content in investigated Al-Cr alloys, as shown in Fig. 2(b), meaning its probable dependence on secondary phase precipitates too.

To conclude this paper we would like to make some comments on the challenging questions from a fundamental point of view and outline future prospects. The TDS technique employed for hydrogen desorption kinetics analysis provides new insight into the hydrogen/microstructure interactions in RS aluminium alloys. It was revealed that hydrogen behaviour in respect to alloy composition is complicated and depends on microstructural features available due to rapid solidification. There appears to be a need for further work that will necessitate a multiscale framework to clarify details of complex hydrogen interactions and evolution in modified RS microstructures related to the topic of hydrogen embrittlement control in high strength aluminium alloys.

\section{Conclusion}

This study has demonstrated the effect both of RSP and $\mathrm{Cr}$ content on hydrogen desorption kinetics from aluminium materials. Based on the above evaluation of the TDS spectra, at least four $\mathrm{H}$ desorption rate peaks in the thermal desorption spectra are identified for Al-Cr alloys. It was found out that studied RS Al-Cr alloys represent the release of hydrogen trapped at $\mathrm{Cr}$ atoms in lattice sites as well as vacancies, dislocations and pores. The most prominent effect is the dominance of the trap state of $\mathrm{Cr}$ atoms in the alloys. The hydrogen amount resided with quenched-in vacancies in $\mathrm{RS}$ aluminum is drastically decreases in the presence of $\mathrm{Cr}$. Furthermore, the amount of hydrogen associated with dislocations increases with rise in $\mathrm{Cr}$ content in the foils. In contrast, along with increase in $\mathrm{Cr}$-rich precipitates the role of pores in hydrogen trapping is decreased in the alloys.

\section{Acknowledgements}

The authors would like to thank Light Metals Education Foundation Inc. and Japan Aluminium Association. This work was supported by Matsumae International Foundation Grant No. 08G11 to I. Tashlykova-Bushkevich.

\section{REFERENCES}

1) P. Novak, R. Yuan, B. P. Somerday, P. Sofronis and R. O. Ritchie: J. Mech. Phys. Solids 58 (2010) 206-226.

2) S. Serebrinsky, E. A. Carter and M. Ortiz: J. Mech. Phys. Solids 52 (2004) 2403-2430
3) G. Lu and E. Kaxiras: Phys. Rev. Lett. 94 (2005) 155501-1-155501-4

4) A. Kamegawa and M. Okada: Rev. High Press. Sci. Technol. 17 (2007) 173-179.

5) T. Inada, I. Chou and H. Eguchi: Ishikawajima Harima Eng. Rev. 45 (2005) 125-129.

6) J. R. Scully, G. A. Young Jr. and S. W. Smith: Mater. Sci. Forum 331337 (2000) 1583-1600.

7) G. Itoh: Proc. 12th Int. Conf. on Aluminium alloys, ed. by S. Kumai, O. Umezawa, Y. Takayama, T. Tsuchida and T. Sato, (The Japan Inst. Light Metals, 2010) pp. 1347-1352.

8) H. Toda, T. Yamaguchi, M. Nakazawa, Y. Aoki, K. Uesugi, Y. Suzuki and M. Kobayashi: Mater. Trans. 51 (2010) 1288-1295.

9) K. Horikawa, H. Yamada and H. Kobayashi: Proc. 12th Int. Conf. on Aluminium alloys, ed. by S. Kumai, O. Umezawa, Y. Takayama, T. Tsuchida and T. Sato, (The Japan Inst. Light Metals, 2010) pp. 371376.

10) L. Katgerman and F. Dom: Mater. Sci. Eng. A 375-377 (2004) 12121216.

11) T. S. Srivatson, T. S. Sudarshan and E. J. Lavernia: Prog. Mater. Sci. 39 (1995) 317-409.

12) Y. Xiao, J. Zhong, W. Li and Z. Ma: J. Mater. Sci. 43 (2008) 324-329.

13) Aluminum: Properties and Physical Metallurgy (Reference Book), ed by J. E. Hatch, (American Society for Metals, Metals Park, Ohio, United States, 1984) pp. 40-63.

14) E. Tal-Gutelmacher, D. Eliezer and E. Abramov: Mater. Sci. Eng. A 445-446 (2007) 625-631.

15) S. Hayashi: Jpn. J. Appl. Phys. 37 (1998) 930-937.

16) I. Tashlykova-Bushkevich, T. Shikagawa, T. Suzuki, V. Shepelevich and G. Itoh: Mater. Sci. Forum 638-642 (2010) 465-468.

17) I. Tashlykova-Bushkevich, T. Shikagawa, V. Shepelevich and G. Itoh: Mater. Sci. Forum 654-656 (2010) 998-1001.

18) G. A. Young Jr. and J. R. Scully: Acta Mater. 46 (1998) 6337-6349.

19) S. I. Miroshnichenko: Quenching from the Liquid State, (Metallurgiya, Moscow, 1982) pp. 1-168 (in Russian).

20) I. Tashlykova-Bushkevich, T. Shikagawa, V. Shepelevich and G. Itoh: Proc. 12th Int. Conf. on Aluminium alloys, ed. by S. Kumai, O. Umezawa, Y. Takayama, T. Tsuchida and T. Sato, (The Japan Inst. Light Metals, 2010) pp. 1791-1794.

21) Information on http://microtm.com.

22) Information on http://escoltd.co.jp.

23) Information on http://www.originlab.com.

24) H. Toda, T. Hidaka, M. Kobayashi, K. Uesugi, A. Takeuchi and K. Horikawa: Acta Mater. 57 (2009) 2277-2290.

25) W. Smith and J. R. Scully: Metall. Trans. A 31A (2000) 179-193.

26) E. J. Lavernia, J. D. Ayers and T. S. Srivatson: Int. Mater. Rev. 37 (1992) 1-44

27) E. Yu. Neumerzhytskaya and V. G. Shepelevich: Perspectivnye Materialy 4 (2005) 69-73 (in Russian).

28) I. I. Tashlykova-Bushkevich: Poverkhnost'. Rentgenovskie, Sinkhrotronnye i Neitronnye Issledovaniya 7 (2010) 105-112 (in Russian).

29) J. Collot: Mater. Manuf. Process. 16 (2001) 595-617.

30) G. Itoh, K. Koyama and M. Kanno: Scr. Mater. 35 (1996) 695-698.

31) T. Izumi and G. Itoh: Proc. 3th Int. Stud. Conf. Ibaraki University, ed. by T. Suzuki (Ibaraki University, 2007) pp. 275-280.

32) H. Toda, K. Minami, K. Koyama, K. Ichitani, M. Kobayashi, K. Uesugi and Y. Suzuki: Acta Mater. 57 (2009) 4391-4403.

33) I. I. Tashlykova-Bushkevich: Proc. 12th Int. Conf. on Aluminium alloys, ed. by S. Kumai, O. Umezawa, Y. Takayama, T. Tsuchida and T. Sato, (The Japan Inst. Light Metals, 2010) pp. 1800-1805.

34) M. de Haas and J. Th. M. de Hosson: Scr. Mater. 44 (2001) 281-286. 